\title{
Stretching the limits of knowledge attainable with live cell imaging in ARTs
}

\author{
David F. Albertini ${ }^{1}$
}

Published online: 23 March 2016

(C) Springer Science+Business Media New York 2016

The history of embryology owes much to the observational skills of pioneering scientists armed with little more than an insatiable curiosity and a good microscope. And true to form, our current ART principles upon which we rely in judging the developmental competencies of embryos produced for treating patients desirous of term pregnancies remain vested in what the embryologist is beholden to under the microscope. Methods like polarization microscopy were first developed as a non-invasive tool, delivering properties of molecular order within oocytes and embryos as signposts of viability and developmental potential, coordinated in time with the introduction of ICSI and assuring a generation of egg-prickers that the meiotic spindle would become an innocent and unharmed bystander after their intrusive acts.

In more recent years, the experimental utility of time-lapse microscopy has been transformed into a pliable diagnostic methodology of its own. The melding of digital imaging, light-emitting diodes of harmless wavelengths, and the invasion of incubators by a generation of rather odd-looking microscopes has conspired to add the element of time to the morphological criteria upon which only snapshots and inferences were stitched into a projection of embryo quality. It is daunting to think that today, the very basic events of pronuclear migration (see cover this month), compaction, blastocoel

Capsule Two recent papers further illustrate the power of live cell microscopy as it applies to the evaluation of mammalian embryo dynamics, bringing answers to long-sought-after fundamental developmental questions and the reality of non-invasive imaging a step closer to practical implementation.

David F. Albertini

DALBERTINI@kumc.edu

1 University of Kansas Medical Center, Kansas, KS, USA expansion, and hatching are all captured for every patient that enters a clinic using time-lapse microscopy and will for themselves and their caregivers reveal these processes over an adjusted time scale that the human brain can process and appreciate for its intrinsic elegance and simplicity.

Speaking for a generation of scientists spawned well before the era of molecular biology, the field of cell biology emerged from a reductionist desire to see more with microscopes and someday piece together a map of interactions between cell organelles and macromolecular complexes that would open avenues for research staged to someday eradicate and/or ameliorate disease. For cell biologists studying the process of reproduction, this was a long and winding road laced with obstacles posed by the very morphological nature of gametes and embryos - too small, too few, too sensitive, and too finicky for imaging what was really going on inside!

With the recent publication of two papers, each exposing the inner workings of pre-implantation mammalian embryos with unique perspectives, the time has come to prepare ourselves for a revolutionary change in how we think about early development and the ART practices of today.

The first publication of note comes from the group of Jan Ellenberg at the EMBO laboratories in Heidelberg (Strnad P et al. Inverted light-sheet microscope for imaging mouse preimplantation development. Nature Methods 2016;13:139142. doi: $10.1038 /$ nmeth.3690). This is a must-read for anyone who has pondered how it is that a one-cell embryo is transformed into a blastocyst comprised of distinct lineages that will propagate the placenta and embryo proper-well known to most as the lineage allocation conundrum. Strnad and colleagues established a technological tour de force in designing a microscope capable of capturing every cell division in scores of mouse embryos, which armed with powerful image analysis and processing tools, yields fate maps of each cell on their way to their appointed position in the fully formed 
blastocyst. From these exhaustive data sets, they can pinpoint that magical moment when fates are assigned (in the mouse at the eight-cell stage of development) and track excursions illuminating the contributions of cell polarity, spindle orientation, and cell repositioning. It seems only a matter of time before this kind of information becomes available for the human embryo, and along with it, the opportunity for clinical embryologists and developmental biologists to have a deeper understanding of these earliest stages of human embryo development.

The second publication we draw attention to aims to identify the relationship between the mechanical properties of 2 pronuclear (2PN) stage embryos, measured by applying negative pressure to the embryo cortex, and viability of embryos, as measured by their ability to form blastocysts (Yanez et al. Human oocyte developmental potential is predicted by mechanical properties within hours after fertilization. Nature Communications. 2016;7:10809, doi: 10.1038/ ncomms10809, www.nature.com/naturecommunications). The study was conducted on 282 mouse and 89 human 2PN stage embryos, the former of which were freshly fertilized and the latter were from previously fertilized and frozen samples that were thawed and cultured for 3-4 h prior to taking measurements. While the thrust of the paper identifies cortical stiffness as a predictor of later embryo viability, an important take-home message elaborated upon by the authors concerns the establishment and regulation of cortical properties over the course of oocyte maturation evidenced by intrin- sic heterogeneity and variability in developmental potential. Buttressing the biophysical dimension of these studies are correlative data sets on transcription profiles and cortical granule release efficiency, all persuasively accounted for as the authors suggest that such an approach "....could be used to add predictive value to current embryo selection techniques...." The importance of this work resides in yet another demonstration of the types of cell biological insights gleaned from advancing technologies designed to continue the quest for diagnostic and non-perturbing methods capable of identifying gametes and embryos that will best serve the needs of our patients.

While science marches forward with a level of sophistication and optimism founded in ground-breaking studies of the likes discussed above, the real-world issues confronting the practice of ARTs are being highlighted by matters of global importance. To this end, we offer our readership an introduction to the Zika virus crisis that in recent weeks has permeated the practice of ARTs (Zika virus infection in semen: a call to action and research. doi: 10.1007/s10815-016-0684-6; The chasm between public health and reproductive research: what history tells us about Zika virus. doi: 10.1007/s10815016-0687-3). And, in continuing to serve the ART community, we hope you enjoy the spectrum of topics in this month's issue ranging from the latest advances in the use of bioreactors for supporting gamete maturation to the influence of blastocyst shrinkage prior to cryopreservation on pregnancy potential. 